\title{
EN TORNO A LA DOBLE NATURALEZA DEL DERECHO DE ROBERT ALEXY
}

\section{ABOUT THE DUAL NATURE OF LAW BY ROBERT ALEXY}

Edgar Cruz Acuña*

\section{RESUMEN}

Se desarrolla el argumento central de la tesis de Robert Alexy sobre la naturaleza dual del Derecho que comprende una dimensión real o fáctica, y otra, ideal o crítica. Dicha tesis es incompatible tanto con el positivismo jurídico excluyente como con el positivismo jurídico incluyente, y con algunas tesis del no-positivismo en su versión extrema. La naturaleza dual del Derecho se expresa y justifica, de un lado, por la fórmula del iusfilósofo Gustav Radbruch que manifiesta que la "injusticia extrema no es Derecho" y, de otro lado, por el argumento de corrección moral. Como consecuencia de este análisis, la definición del Derecho es algo que depende tanto de los hechos sociales como de aquello que el Derecho debe ser.

\section{PALABRAS CLAVE:}

Derecho, moral, positivismo, naturaleza, justicia, corrección moral, coerción, normatividad.

\begin{abstract}
The central argument of Robert Alexy's thesis is developed, concerning the dual nature of Law that includes a real or factual dimension, and another, ideal or critical. This thesis is inconsistent with both the exclusive legal positivism and the inclusive legal positivism, and with some thesis of non-positivism in its extreme version. The dual nature of law expresses and justifies by Gustav Radbruch 's formula which states that the "extreme injustice is not Law" on the one hand, and by the argument of moral correctness on the other. As a result of this analysis, the definition of the Law is something that depends on social facts in what the Law should be.
\end{abstract}

\section{KEY WORDS:}

Law, morality, positivism, nature, justice, moral correctness, coercion, normativity.

\footnotetext{
* Docente de la Universidad Católica Sedes Sapientiae: edgar.cruz@pucp.edu.pe
} 


\section{INTRODUCCIÓN}

En estos últimos decenios, el iusfilósofo alemán Robert Alexy, a través de sus múltiples escritos que pretenden fundamentar y clarificar la esencia del Derecho, representa una voz muy cualificada y una respuesta apropiada a la cuestión. Sitúa su teoría de la doble naturaleza del Derecho, por un lado, en el marco de la Filosofía del Derecho cuya propiedad esencial consiste en ser una rama de la Filosofía que reflexiona sobre el fenómeno jurídico y, por otro lado, en diálogo con las conocidas tesis del positivismo jurídico excluyente e incluyente, resaltando las diferencias existentes entre estas concepciones y su visión del no-positivismo jurídico incluyente moderado, y demostrando por qué esta última visión de Derecho ofrece mayores garantías y mejores posibilidades de fundamentación teórica y práctica.

En este sentido, Alexy desarrolla las bases no sólo de una teoría general del Derecho sino también de una metateoría del Derecho, es decir, de una reflexión acerca de la teoría general del Derecho.

La explicación de la naturaleza del Derecho y su consecuente formulación conceptual ha sido, desde la antigüedad, un problema central de la Filosofía del Derecho que, según el profesor alemán, tiene por objeto específico dar respuesta a la pregunta ontológica fundamental de lo jurídico, es decir, ¿qué es el Derecho? Es así que, en sus numerosos escritos, que desde 1978 el autor viene publicando regularmente, así como en sus múltiples intervenciones como profesor y conferencista internacional, clarifica y desarrolla con amplitud las tesis más emblemáticas de su teoría jurídica, situándolas en el marco de la discusión actual sobre el concepto del Derecho.

Este estudio introductorio al aspecto central del pensamiento de R. Alexy, se propone cuatro objetivos concretos que se desarrollarán, respectivamente, en tres secciones propuestas. En la primera sección se presenta una breve reseña biográfica de Robert Alexy, que nos ayudará a disponer de una comprensión mayor de su pensamiento y del contexto del trabajo de este genuino iusfilósofo. En la segunda sección se analiza brevemente las principales tesis sobre el concepto y la naturaleza del Derecho. Y, por último, la tercera sistematiza las más importantes y diversas objeciones que se ha formulado a la tesis en mención.

\section{Breve Reseña Biográfica de RobertAlexy}

Robert Alexy es un iusfilósofo alemán, catedrático de Derecho Público y Filosofía del Derecho en la Universidad de Kiel. Nació el 9 de setiembre de 1945 en Oldenburg (Alemania). Después de culminar la educación secundaria, sirvió durante tres años en el Ejército Federal Alemán, en el cual terminó con el rango de teniente. Estudió Derecho y Filosofía en la Universidad de Georg-Ausgus de Gottingen.

Después de superar el primer examen del Estado para culminar la licenciatura de Derecho en 1973, trabajó hasta 1976 en su tesis sobre "Teoría de la argumentación jurídica". Durante este tiempo, su actividad de investigación recibió financiación de la fundación Studienstiftung des deustschen Volkes. En 1982 recibió el premio de la Academia de la Ciencia de Gottingen, en la categoría de Filosofía e Historia, por la investigación conducente a la Teoría de la argumentación jurídica, publicada por 
primera vez en el año 1978. En este mismo año culminó su práctica jurídica luego de superar el segundo examen del Estado. En adelante, hasta 1984, se desempeñó como asistente de cátedra del profesor Ralf Dreier en el Departamento de Teoría General del Derecho en Gottingen. En 1984 culminó su habilitación en Derecho Público y Filosofía del Derecho en la Facultad de Derecho de la Universidad de Gottingen con su obra "Teoría de los derechos fundamentales".

Entre 1994 y 1998 fue presidente de la sección alemana de la Asociación Internacional de Filosofía del Derecho y Filosofía Social (IVR). En 1992 publicó su obra "El concepto y la validez del Derecho", que representa la obra comprensiva de su teoría del Derecho. Desde el año 2002 es miembro de la Academia de la Ciencia de Gottingen en la categoría de Filosofía e Historia.

Entre sus obras principales destacamos las siguientes:

- Teoría de la Argumentación Jurídica: Teoría del discurso racional como teoría de la fundamentación jurídica (1983). Traducido por el Dr. Manuel Atienza e Isabel Espejo, Madrid: Centro de Estudios Constitucionales, 1989.

- Teoría de los derechos fundamentales (1985; segunda edición 1994). Traducida por Ernesto Garzón Valdés, Madrid: Centro de Estudios Constitucionales, 1993.

- La doble dimensión del Derecho. Traducido por Carlos Bernal Pulido. Lima; Palestra Editores, 2011.

- Traducido por Carlos Bernal Pulido como "El concepto y la naturaleza del Derecho", Madrid: Marcial Pons, 2008.

- El concepto y la validez del Derecho. Traducido por Jorge Fabras, Madrid: Gedisa editores, 2008.

\section{Principales Tesis de Robert Alexy sobre el Concepto y la Naturaleza del Derecho}

La Filosofía del Derecho es una disciplina similar a la Meta-ética, pero con un objeto distinto. Su objeto de estudio no es la Ontología ni la Moral ni la Epistemología, sino el Derecho. En este sentido, la diferencia entre la Dogmática Jurídica y la Filosofía del Derecho parece ser análoga a la diferencia que existe entre la Teoría moral normativa y la Metaética. La primera se ocupa de problemas morales clásicos como aquellos a los que se refieren las preguntas: ¿Qué es lo debido desde el punto de vista moral? ¿Qué tipo de acciones son lícitas o ilícitas? ¿Qué diferencia hay entre actos del hombre y actos humanos? ¿Qué tipo de personas debemos ser? ¿Cuáles son las virtudes morales? ¿Qué son los valores éticos? ¿Qué es aquello que exige la justicia? ¿Qué es la justicia? O ¿Cómo debemos vivir nuestra vida? Responder a alguna de estas preguntas implica llevar a cabo afirmaciones de tipo moral o afirmaciones acerca de lo que es válido desde el punto de vista moral.

A diferencia de ello, la Metaética se ocupa de cuestiones relativas a las afirmaciones de tipo moral que se hacen en el ámbito de la Teoría moral normativa. Se pregunta, por ejemplo, si existen valores morales o si lo que existe solo es una amalgama de sentimientos y de actitudes humanas acerca de los problemas morales, pero no existe ninguna propiedad que pueda indicar si dichos sentimientos o dichas actitudes son correctas o incorrectas. La Metaética se ocupa del problema de si existen propiedades morales que permitan decidir acerca de la corrección o falta de corrección de las afirmaciones que se hacen en el ámbito de la Teoría moral normativa y de si es posible el 
conocimiento moral o justificar las afirmaciones de contenido moral. En consecuencia, mientras la Teoría moral normativa implica emitir juicios de primer orden, la Metaética implica emitir juicios de segundo orden, es decir, juicios acerca de los juicios de primer orden.

Una relación análoga es la que existe entre la Dogmática jurídica y la Filosofía del Derecho. La primera se ocupa, esencialmente, de la pregunta sobre qué es lo válido desde el punto de vista jurídico, y de preguntas como cuál es la solución que el Derecho otorga a cada caso concreto, o cuáles son las acciones jurídicamente permitidas, prohibidas u ordenadas. Por su parte, la Filosofía del Derecho se ocupa de cuestiones cuya respuesta es a veces necesaria para responder los interrogantes que se proponen en el ámbito de la dogmática jurídica. La Filosofía del Derecho se pregunta, por ejemplo: ¿En qué tipo de entidades consiste el Derecho? ¿Qué es aquello que las conecta para hacerlas formar el todo llamado Derecho? ¿Cuáles son las propiedades que caracterizan a cada una de estas entidades y al todo que denominamos Derecho? Las respuestas a estas preguntas se expresan mediante juicios de segundo orden, es decir, juicios acerca de los juicios que se expresan en la Dogmática jurídica.

Ahora bien, es posible incluso pensar en un tercer nivel de reflexión, en el que en ocasiones se sitúa la reflexión de Alexy. Se trata del ámbito de la Metateoría del Derecho. Las reflexiones en este ámbito se expresan por medio de juicios de tercer orden, es decir, juicios acerca de los juicios que se emiten en el ámbito de la Filosofía del Derecho. Así, la Metateoría del Derecho se ocupa de preguntas tales como: ¿Qué tipo de problemas son aquellos que debe afrontar la Filosofía del Derecho? ¿Qué tipo de argumentos se utilizan para definir en qué tipo de entidades consiste el Derecho y qué es aquello que las vincula para formar el todo llamado Derecho? O ¿Cómo es posible evaluar la corrección de las conclusiones que derivan de estos argumentos y de las justificaciones que estos argumentos representan? Si la Filosofía del Derecho es una reflexión acerca de la naturaleza del Derecho, la Metateoría del Derecho es una reflexión acerca de la reflexión sobre la naturaleza del Derecho.

Con base a este marco conceptual es posible entender cuáles son las principales ideas que Alexy expone acerca de la Filosofía del Derecho, de la Metateoría del Derecho y del concepto y la naturaleza del Derecho.

\subsection{Tesis relativas a la Filosofía del Derecho}

Las principales ideas que Alexy defiende en relación con la Filosofía del Derecho son las siguientes: a) la Filosofía del Derecho como una rama de la Filosofía; b) el método de la Filosofía del Derecho como el análisis sistemático de los argumentos sobre la naturaleza del Derecho; c) la tesis de la naturaleza general; d) la tesis del carácter específico; e) la tesis de la relación especial; f) la tesis del ideal comparativo; y, g) la tesis de las tres dimensiones de la Filosofía del Derecho.

\subsubsection{La Filosofía del Derecho es una rama de la Filosofía}

La Filosofía del Derecho es una rama de la Filosofía. Ésta es la reflexión general y sistemática sobre la realidad existente, lo que debe hacer o es bueno, y sobre cómo es posible el conocimiento acerca de estas dos cosas. Esta definición, según el autor, enuncia las principales ramas 
de la Filosofía, es decir, la Ontología o Metafísica, la Ética o Moral y la Teoría del Conocimiento o Gnoseología. La Filosofía del Derecho se plantea estas mismas preguntas pero con relación al Derecho mismo. Plantearse estas preguntas en relación con el Derecho es lo que define a la Filosofía del Derecho como razonamiento general y sistemático acerca de la naturaleza del Derecho.

\subsubsection{El método de la Filosofía del Derecho}

El método de la Filosofía del Derecho es el análisis sistemático de los argumentos acerca de la naturaleza del Derecho. A fin de responder las preguntas acerca de lo que existe, lo que debe hacerse jurídicamente, y de cómo es posible el conocimiento acerca de estas dos cosas, la Filosofía del Derecho parte de un estudio de las diferentes precomprensiones del Derecho y de sus relaciones con las propiedades del fenómeno jurídico. Esto se lleva a cabo mediante el análisis sistemático de los argumentos expuestos en la discusión sobre la naturaleza del Derecho. Tales argumentos se refieren a tres problemas:

a. ¿̇en qué clase de entidades consiste el Derecho, y cómo están conectadas estas entidades de tal modo que conforman la entidad global que llamamos Derecho?;

b. ¿cómo debe entenderse las propiedades que caracterizan la dimensión real o fáctica del Derecho, en particular, la expedición autoritativa y la eficacia social?; y

c. ¿cómo debe entenderse las propiedades que caracterizan la dimensión ideal o crítica del Derecho, en particular, la corrección - legitimidad del Derecho y la relación entre el Derecho y la Moral?
En relación con estos problemas, Alexy defiende cuatro tesis: de la naturaleza general, del carácter específico, de la relación especial y del ideal comprensivo.

\subsubsection{Tesis de la naturaleza general}

Esta tesis señala que todos los problemas de la Filosofía pueden presentarse en la Filosofía del Derecho.

\subsubsection{Tesis del carácter específico}

Esta tesis sostiene que existen problemas específicos de la Filosofía del Derecho. Estos se deben al carácter específico del Derecho, que para el autor está determinado por su doble naturaleza, es decir, que el Derecho tiene dos dimensiones: la dimensión real o fáctica y la dimensión ideal o crítica.

\subsubsection{Tesis de la relación especial}

Esta tesis refiere que existe una relación especial entre la Filosofía del Derecho y las otras áreas de la Filosofía práctica, especialmente con la Moral y la Política. El fundamento de esta tesis está ligado a las ideas que el autor defiende en la Teoría de la argumentación jurídica (Alexy, 1878). La tesis del caso especial señala que el discurso jurídico es un caso especial del discurso práctico general. $\mathrm{Si}$ esto es así, es lógico que la disciplina que estudia el discurso jurídico (la Filosofía del Derecho) esté también en una relación especial con la Filosofía práctica, es decir, con la disciplina que estudia el discurso práctico general.

\subsubsection{Tesis del ideal comprensivo}

Sostiene que la Filosofía del Derecho puede tener éxito únicamente si se aceptan tres tesis relativas de la naturaleza general, el carácter especial y la relación especial. Lo contrario al ideal 
comprensivo es un máximo restrictivo, que sostiene que la Filosofía del Derecho no debe implicarse en problemas genuinamente filosóficos, que debe delegar a la filosofía práctica la solución de todos los problemas normativos y se concentrara exclusivamente al estudio de la dimensión real o fáctica del Derecho, la cual se expresa en su carácter institucional o autoritativo.

Por el contrario, Alexy sostiene que la Filosofía del Derecho debe plantearse problemas genuinamente filosóficos en relación con el Derecho, así como preguntas normativas, y que, junto a la explicación de la dimensión real o fáctica del Derecho debe explicar también la dimensión ideal o crítica del mismo, referido a la corrección moral o a la legitimidad del Derecho.

\subsubsection{Tres dimensiones de la Filosofía del Derecho}

Las tres dimensiones de la Filosofía del Derecho son las siguientes: filosófica, técnica y crítica. De estas tres, la dimensión filosófica se refiere al conocimiento reflexivo general y sistemático que es propio de la Filosofía del Derecho; la dimensión técnica tiene que ver con la clarificación de las instituciones y conceptos jurídicos, la arquitectura del sistema jurídico y la estructura de la argumentación jurídica; finalmente, la dimensión crítica se relaciona con el perfeccionamiento del Derecho positivo mediante la crítica filosófica.

\subsection{Tesis relativa a la Metateoría del Derecho}

\subsubsection{Naturaleza genuinamente filosófica de algunos de los argumentos acerca de la naturaleza del Derecho}

Alexy sostiene que algunos de los argumentos que forman parte del debate acerca de la naturaleza del Derecho tienen una naturaleza genuinamente filosófica. Este tipo de argumentos tienen que ver con la cuestión de si es posible reducir el Derecho a fenómenos pertenecientes al reino físico o al reino psíquico, o si, por el contrario, como lo sugiere Hans Kelsen, las normas jurídicas, en cuanto proposiciones prescriptivas pertenecen a un tercer reino especial, en el sentido del discurso de Gottlob Frege.

Como es sabido, G. Frege sostiene que los sentidos de las palabras tienen una existencia objetiva propia. Son, de este modo, como los animales, los árboles o las cosas. Su existencia no depende de la mente humana, sino que pertenecen a un tercer reino que compone el mundo, junto al reino mental o psíquico y al reino físico. Los significados de las palabras ciertamente no son entidades físicas, pero tampoco dependen de la mente de las personas. Por ejemplo, la proposición "el cielo está nublado" tiene un valor de verdad, y por tanto, es verdadera o falsa, con prescindencia de lo que alguien piense de ella.

Lo que Alexy sostiene es que para determinar si el Derecho puede reducirse a simples fenómenos físicos o mentales, o es una entidad objetiva irreductible, perteneciente a este tercer reino, es necesario afrontar el problema genuinamente filosófico de si en verdad existe un tercer reino como aquel del que habla Frege.

\subsubsection{Naturaleza conceptual de algunos argumentos acerca de la naturaleza del Derecho}

Según Alexy, algunos argumentos relativos a la naturaleza del Derecho tienen un carácter conceptual. En este 
sentido, comparte la tesis del británico John Austin (1911 1960) que sostiene que los argumentos conceptuales no pueden constituir la última sino la primera palabra de un análisis acerca de la naturaleza de una cosa. Pone como ejemplo el argumento a favor de considerar a la coerción como una propiedad esencial del Derecho. Según esto, el concepto de Derecho, tal como se usa en el presente, exige por lo menos que algunas de normas del sistema jurídico sean exigibles coactivamente y que la coerción, por lo menos algunas veces y para algunas personas, pueda ser una motivación para obedecer el Derecho.

Otro argumento conceptual que propone Alexy en su teoría del Derecho es el argumento de la corrección, el mismo que se desarrolla en dos pasos: en el primero, se intenta mostrar que el Derecho eleva necesariamente una pretensión de corrección moral; el segundo, consiste en explicar que esta pretensión implica una conexión necesaria entre el Derecho y la Moral.

Según la pretensión de corrección, uno de los presupuestos necesarios de los conceptos es que pretenden ser correctos y aceptados como tales por parte de la comunidad jurídica y la comunidad en general. Ahora bien, esta pretensión implica una conexión necesaria entre el Derecho y la Moral porque, cuando las razones provenientes del Derecho positivo no son suficientes para resolver los casos concretos, especialmente los difíciles, la pretensión de corrección permite recurrir a otras razones, entre ellas: principios generales y normas morales. Sin embargo, esta misma pretensión otorga prioridad a las razones de justicia sobre todas las otras razones para resolver tales casos concretos.
La conclusión de Alexy es definitiva: "Esto ya es suficiente para establecer que la pretensión de corrección necesariamente se refiere al razonamiento moral cuando la decisión no puede adoptarse solo con base en razones procedentes del Derecho positivo", o, expresado en términos más concretos: "La pretensión de corrección no sólo implica el poder jurídico del juez para aplicar razones morales en los casos difíciles; también implica la obligación jurídica de hacerlo cuando sea posible".

\subsubsection{Naturaleza de algunos argumentos acerca de la naturaleza del Derecho.}

Según el profesor de Kiel, otros argumentos acerca de la naturaleza del Derecho tienen un carácter práctico o normativo. Dichos argumentos son relevantes, por ejemplo, cuando se alude que el Derecho como tal o algunas propiedades del mismo son necesarias para llevar a cabo ciertas funciones o para satisfacer las exigencias de ciertos valores. Un argumento de esta naturaleza es aquel que señala que la coerción es una propiedad esencial del Derecho porque es necesaria para que el Derecho cumpla con los valores de la certeza y la eficiencia que le son inherentes. Otro argumento práctico o normativo que utiliza en su Teoría del Derecho es el argumento de la injusticia extrema de Gustav Radbruch.

\subsection{Tesis relativas al concepto y naturaleza del Derecho}

\subsubsection{Tesis de la naturaleza dual}

Esta tesis expresa que el Derecho necesariamente implica dos dimensiones: una dimensión real o fáctica y una dimensión ideal o crítica. 


\subsubsection{Incompatibilidad de la tesis de la naturaleza dual.}

Sostiene que la tesis de la naturaleza dual es incompatible tanto con el positivismo jurídico exclusivo como con el positivismo jurídico inclusivo. También, la tesis es incompatible con algunas variantes del no-positivismo jurídico, como el nopositivismo exclusivo que sostiene que la validez jurídica desaparece en todos los casos en que existe un defecto moral, o como el no-positivismo inclusivo extremo que propone que la validez jurídica no se afecta de ninguna manera a causa de los defectos morales.

Según la tesis del iusfilósofo, el positivismo jurídico exclusivo, al excluir toda posibilidad de incorporar argumentos morales al Derecho, porque dichos argumentos son razones no autoritativas y el Derecho solo comprende razones autoritativas, elude la existencia de una dimensión ideal o crítica en el Derecho y, por eso, es incompatible con la tesis de la naturaleza dual del Derecho.

También el positivismo jurídico inclusivo resulta incompatible con esta tesis, pues sostiene que la incorporación de tales argumentos no es necesaria sino solo aleatoria y contingente. Es incompatible con la tesis dual porque implicaría que no sería necesario sino solamente posible que el Derecho tuviese una dimensión ideal o crítica vinculada con la corrección moral.

Por su parte, de acuerdo con el nopositivismo jurídico, los argumentos morales que son indispensables para dar una respuesta bien fundada a interrogantes jurídicos se incorporan necesariamente al Derecho. Por eso, el no-positivismo resulta compatible con la tesis de la naturaleza dual.
Pero no son compatibles con esta tesis algunas variantes del nopositivismo jurídico extremos que rehúyen la existencia de la dimensión fáctica o real del Derecho. Así, por ejemplo, el no-positivismo exclusivo radical sostiene que los defectos morales siempre tienen como efecto la pérdida de la validez jurídica. Esta versión del nopositivismo es incompatible con la tesis de la naturaleza dual porque somete por completo la dimensión fáctica o real del Derecho a la dimensión crítica o ideal, de tal manera que permite que esta desplace por completo a aquella. Por su parte, el no-positivismo inclusivo extremo o superinclusivo sostiene que a pesar de existir una conexión conceptual necesaria entre el Derecho y la Moral, los defectos morales nunca repercuten en una pérdida de validez jurídica. Esta versión expresa un sesgo injustificado a favor de la dimensión fáctica o real del Derecho con desmedro a la dimensión crítica o ideal del Derecho.

Alexy sostiene que su tesis del nopositivismo inclusivo, que acepta la fórmula de G. Radbruch, de acuerdo con la cual solo cuando el Derecho es extremadamente injusto pierde su validez, sí resulta compatible con la tesis de la naturaleza dual del Derecho.

\subsubsection{Las propiedades esenciales del Derecho}

La pregunta filosófica acerca de la naturaleza de una cosa, indaga acerca de las propiedades esenciales de esta cosa. Si se pretende responder a esta cuestión en lo que se refiere al Derecho, es decir, cuáles son sus propiedades esenciales, debe explorarse cuáles son las propiedades que integran de ambas dimensiones del Derecho: real o fáctica e ideal o crítica. Estas propiedades son inherentes a la naturaleza del 
Derecho donde quiera que exista, independientemente de las personas y las culturas. Por esta razón, también repercuten en la definición del Derecho. Según Alexy, en la dimensión real o fáctica se hallan tres elementos que son fundamentales. El primero es la relación entre el Derecho y la coerción o la fuerza; el segundo, la relación entre el Derecho y la institucionalización de los procedimientos de creación y aplicación de normas; y el tercero, la relación entre el Derecho y el asentimiento o aceptación real acerca del mismo. De estos tres elementos, el profesor de Kiel, realiza un análisis más amplio de la coerción o la fuerza como propiedad esencial del Derecho.

Por otro lado, sostiene que la propiedad esencial del Derecho en su dimensión ideal o crítica es la corrección. Esta propiedad tiene que ver con la relación necesaria entre el Derecho y la Moral.

\subsubsection{La coerción como propiedad esencial del Derecho}

Según el autor, varios argumentos jurídicos justifican que la coerción es una propiedad esencial del Derecho. Uno de ellos es el argumento conceptual que ya se ha mencionado. Sin embargo, el argumento de mayor peso es el práctico normativo que sostiene que la coerción es un medio imprescindible para que el Derecho pueda cumplir las funciones que le imponen dos valores con los cuales el Derecho tiene una conexión necesaria, es decir, los valores de la certeza y de la eficacia jurídica.

\subsubsection{La corrección como propiedad esencial del Derecho}

Desde la perspectiva del autor, dos propiedades esenciales del Derecho, que garantizan a la corrección como una propiedad esencial del mismo en su dimensión ideal o crítica, es que el Derecho eleva una pretensión de corrección y que, de acuerdo con la fórmula de Radbruch, la injusticia extrema no es Derecho.

\section{A modo de Conclusión: Algunas Apreciaciones Críticas a las Tesis de Alexy}

Algunas de las tesis defendidas por Robert Alexy han sido materia de crítica por parte de connotados iusfilósofos. Posiblemente las tesis más criticadas hayan sido aquellas acerca de la existencia esencia de una dimensión ideal o crítica del Derecho, y a los elementos fundamentales que componen esta dimensión, es decir, la pretensión de corrección moral y la aceptación de la fórmula de Radbruch, según la cual, la injusticia extrema no es Derecho. En este sentido, son conocidas las objeciones formuladas por Eugenio Bulygin y que, con las réplicas de Alexy, se publicaron en el texto: La pretensión de corrección del Derecho. La polémica sobre la relación entre Derecho y Moral (2001).

Asimismo, desde el mundo anglosajón se han formulado las críticas a la tesis contenida en la fórmula de Radbruch. Así, por ejemplo, pueden mencionarse la crítica de Brian Bix, según la cual, la mencionada fórmula se entiende sin problema alguno como una orientación jurídica y moral dirigida al juez, de acuerdo con la cual el Juez debe abstenerse de aplicar las normas extremadamente injustas que componen un sistema jurídico perverso. Pero Bix refuta que de esta fórmula se deriven algunas consecuencias para las teorías acerca de la naturaleza del Derecho. En este 
sentido, la fórmula solamente sería una directriz que establece que el juez no puede aplicar un Derecho que sea extremadamente injusto, pero nunca la prueba de que exista una conexión conceptual necesaria entre el Derecho y la Moral.

Otra de las críticas más prominentes que se ha dirigido a la teoría de Alexy acerca del concepto y la naturaleza del Derecho en los últimos años proviene de Joseph Raz, en su texto "El argumento de la injusticia, o sobre cómo objetar el positivismo jurídico (2007). Raz sostiene que Alexy no identifica el positivismo jurídico correctamente; que para determinar lo que es y debe ser el Derecho no se necesita de consideraciones de tipo moral; que el Derecho sí eleva una pretensión de corrección pero esta no es una pretensión de corrección moral, sino un obvio presupuesto lingüístico de todo acto de habla, y que la fórmula de Radbruch no es inconsistente de ninguna manera con el concepto del positivismo jurídico, pues esta doctrina entiende que las propias fuentes del Derecho prohíben al juez aplicar normas jurídicas que sean extremadamente injustas.

Ante esta y otras críticas que se ha planteado en torno la tesis de Alexy, cabe señalar que el profesor no presenta una teoría del Derecho acabada, que cumpla con las exigencias supremas que su propio proyecto acerca de la Filosofía del Derecho traza, sino que de manera orgánica y sistemática expone una temática a desarrollar y sobre el cual hace falta aún mucho que decir.

\section{REFERENCIAS:}

ALEXY, R. (2008) El concepto y la naturaleza del derecho; traducción y estudio introductorio de Carlos Pulido. Madrid: Marcial Pons.

ALEXY, R. (2007) Teoría de la argumentación jurídica: la teoría del discurso racional como teoría de la fundamentación jurídica. Lima: Palestra.

ALEXY, R (2001) La pretensión de corrección del derecho: la polémica Alexy/Bulygin sobre la relación entre derecho y moral.Bogotá: Universidad Externado de Colombia.

ALEXY, R. (1998) Derecho y moral: ensayos sobre un debate contemporáneo. Rodolfo Vázquez (ed), con contribuciones de Robert Alexy... [et.al.]. Barcelona: Gedisa.

ALEXY, R. (1995) Teoría de los derechos fundamentales. Traducción e introducción de Luis Villar Borda. Bogotá: Universidad Externado de Colombia.

GARCÍA FIGUEROA, A. (1998) Principios y positivismo jurídico: el no positivismo principialista en las teorías de Ronald Dworkin y Robert Alexy / Alfonso Jaime García Figueroa. Madrid: Centro de Estudios Políticos y Constitucionales.

BERNAL PULIDO, C. (Ed) (2011) La doble dimensión del Derecho: autoridad y razón en la obra de Robert Alexy / Carlos Bernal Pulido, editor... [et all. Lima: Palestra. 